REVISTA DE DERECHO UNED, NÚM. 11, 2012

\title{
RESPONSABILIDAD TAXONÓMICA EN LA HISTORIA CLÍNICA
}

\author{
RESPONSABILITY IN THE TAXONOMY OF THE PATIENT \\ SUMMARY
}

Miren Tamayo Vivanco

Doctoranda de la Facultad de Derecho y la ETSII y de IT de la UNED, Madrid

Resumen: El compromiso de separar las competencias que proponen, literalmente, por una parte un paciente y por otra, un e-técnico ante la observación de una dato clínico concluye con la exposición, al menos, de dos grupos taxonómicos . La necesidad de cada taxonomía conduce a actuar de un modo muy diferente con el mismo dato de modo que la legislación que origina en el primer grupo desarrolla la «Summary Patient», y en el segundo grupo empuja a incrementar la generación de auditorías dentro y fuera de cada unidad de sección de la Administración Electrónica. Finalmente, se recogen aquellos principios que parecen contemplar estas consideraciones en relación exclusiva al Consentimiento Informado.

Palabras claves: taxonomía, agente, privacidad, grupo taxonómico, derechos ARCO, seguridad del paciente, incidencia, consentimiento informado, historia clínica.

Abstract: The difficulty to split the competences between the perspective of, literally, a patient, and an informatic e-technician on the observance of a clinical data concludes with the definition of two, at least, taxonomic groups. The necesity of each taxonomy compelles to act in a very different way with the same data and produces such a legislation that on the first group developes the «Summary Patient», and on the second one pushes to increase the management of auditories 
inside and outside the unit of the e-section of the Electronic Administration. In exclusive relationship with the informed consentment are compilled that foundations that seem to consider the proposed view.

Keywords: taxonomy, agent, privacy, taxonomic group, ARCO rights, summary patient, incidence, informed consent, health and medical record.

Recepción original: 02/07/2012

Aceptación original: 27/07/2012

Sumario: I. Premisas de la Taxonomía; II. Grupos Taxonómicos; III. Consideración del Consentimiento Informado.

\section{PREMISAS DE LA TAXONOMÍA}

Del reconocimiento de la importancia del desarrollo de las Taxonomías, podemos extraer algunos principios o consideraciones generales que nos pueden servir a modo de guía previo a exponer el discurso vigente acerca de las responsabilidades tanto desde el campo legal como desde el técnico en el área de tratamiento de la Historia Clínica:

- resaltando la importancia de los agentes ${ }^{1}$ identificados en cada época en torno a la percepción y tratamiento de la privacidad y su posterior consideración desde un estado inicial de bienestar, y ampliando el campo de observación cuando el ciudadano hubiera alcanzado el horizonte de paciente resulta factible detectar más fácilmente aquellas situaciones por las que debe discurrir el desarrollo del dogma legislativo, la labor del legislador y la de un supervisor técnico que adoptará medidas de salvaguarda a fin de sobrellevar posibles incidencias en el tratamiento de estos especialísimos datos de carácter personal

- a fin de poder detectar aquellos posibles Niveles de Intimidad que el individuo considera equilibrio entre su percepción, anteriormente descrita, y la consiguiente manipulación del dato clínico no se deberá olvidar, sino más bien registrar aquellos escenarios y contextos en los que se extraen estos significados

1 «Agent Jurisprudence». Michael N. Huhns, Munindar P. Singh. IEEE Internet Computing. March - April 1998. http:/computer.org/internet (Acceso URL: Julio 2012). «A Formal Model of Legal Argumentation». Giovanni Sartor. 
- con independencia del status quo en el que se encontrara el ciudadano, éste debiera preguntarse en qué medida se es consciente de lo que ocurre, bien fuera en relación al Gobierno, a la familia, al Empleo, a las políticas de cada comunidad, etc.

- Reconociendo la Naturaleza de la Información y de cómo se crean intereses sobre ella, resultará factible superar la perspectiva de cómo cada Sociedad protege los problemas en torno a la Intimidad Personal frente al denominado «bien social»

- se puede concluir que cada Taxonomía debiera decir más bien poco más allá de su propio contexto

- advertencia de los posibles peligros, para cada Grupo $\mathrm{Hu}$ mano, que participe en la manipulación del dato médico del posible Uso Secundario que se le pudiera dar

- de modo genérico se identifican tres grandes grupos en torno a lo que se ha venido en llamar estructura de la e-Sanidad: el propio paciente, el técnico más o menos caracterizado en su profesión y que debe evitar la perdida de información; y, la del asistente sanitario no informático que en el día a día interacciona y trata la salud del paciente

- para el reconocimiento de principios y agentes debemos mantener continuamente activo el Ciclo de Deming ${ }^{2}$ o «Plan. Do.Check.Act»

- reconociendo la necesariedad de la actualización y colaboración en la elaboración de los Códigos de Buenas Prácticas ${ }^{3}$, así como de la importancia de recabar adecuadamente aquellos Requerimientos Informados ${ }^{4}$ correspondientes a cada Sistema Nacional de Salud cada Grupo Humano de Taxonomía que participa en el soporte de la e-Salud ${ }^{5}$ detectará con mayor nitidez aquellas

2 ISO/IEC 27001.

3 Código de Buenas Prácticas Clínicas. CPMP/ICH/135/95, (pág. 18)

http://www.ema.europa.eu/docs/en_GB/document_library/Scientific_guidel ine/2009/09/WC500002874.pdf (Acceso URL: Agosto 2012).

${ }^{4}$ Jurisprudencia de Telecomunicaciones. Editorial Aranzadi. Coleccion: Jurisprudencia Comentada. Cap IV. II. «Naturaleza Jurídica de los Requerimientos de Información». 2008 (pág. 250).

${ }^{5} \mathrm{La}$ OMS define la e-health como el uso en el sector de la salud de aquella información digital, transmitida, almacenada u obtenida electrónicamente para el apoyo al cuidado de la salud tanto a nivel local como a distancia. No se fabula si se decide que la e-health salva vidas, dado que permite el envío de datos online vitales en situaciones donde unos pocos minutos son los que separan a un paciente de la vida a la muerte.Existe un Observatorio global de la e-heatlh conocido como Goe, nacido a principios de 2005 y que publica un informe anual, así como directrices dirijidas a los países interesados. 
entradas y salidas para los activos que hubiera que proteger o vigilar dentro de su Grupo. Es de este modo, como el Tecnólogo sabe de antemano que nunca es un plus el manejo y aplicación de estándares reconocidos internacionalmente.

A modo de ejemplo, exponemos algunos de los esquemas que resulta factible deducir sobre las directrices anteriormente expuestas:

\section{GRUPOS TAXONÓMICOS}

\section{Tabla 1. Taxonomía del Paciente}

\begin{tabular}{|c|l|}
\hline $\begin{array}{c}\text { Percepción del modo en cómo } \\
\text { se recopila su PII', Personal } \\
\text { Informatic Information }\end{array}$ & se presenta voluntariamente a la consulta y se recopila su in- \\
formación
\end{tabular}

http://www.who.int/kms/initiatives/ehealth/en/ (Acceso URL: octubre 2012)

${ }^{6}$ PII, Personal identificable information

http://en.wikipedia.org/wiki/Personally_identifiable_información (Acceso URL: julio 2012).

${ }^{7}$ LOPDAT (art. 5.1.d), L 41/2002 (art. 18), L 56/2007 (art. 1.d).

${ }^{8}$ Enlaces. http://formacion.seguridaddelpaciente.es/Enlaces.aspx

http://www.who.int/patientsafety/es/ (Acceso URL: Julio 2012). 


\section{Tabla 2. Taxonomía del e-Técnico}

\begin{tabular}{|c|c|}
\hline $\begin{array}{l}\text { Responsabilidad de } \\
\text { Impermutabilidad de } \\
\text { Información }\end{array}$ & $\begin{array}{l}\text { debe responder de la veracidad de los datos que maneja y que } \\
\text { no experimentan modificaciones }\end{array}$ \\
\hline Colaboración Auditada & $\begin{array}{l}\text { debe poder proporcionar y filtrar en el documento curricu- } \\
\text { lar propuesto aquellos datos de los que con autorización for- } \\
\text { mulada ya aceptada se ejerciera la defensa de los Derechos } \\
\text { ARCO }\end{array}$ \\
\hline $\begin{array}{l}\text { Conocimiento de la } \\
\text { Legislación a la que asiste }\end{array}$ & $\begin{array}{l}\text { debe responder a la dinámica de las Auditorías Internas y Ex- } \\
\text { ternas en materia de protección de datos }\end{array}$ \\
\hline Responsabilidad de Incidencia & $\begin{array}{l}\text { como responsable de un incidente debe, conforme al procedi- } \\
\text { miento establecido en la Política de sus Empresa, comunicar- } \\
\text { lo, y tratarlo minimizando su suceso }\end{array}$ \\
\hline $\begin{array}{l}\text { Aportación de su experiencia } \\
\text { en la Optimización de la } \\
\text { e-Administración }\end{array}$ & $\begin{array}{l}\text { debe cooperar en el tratamiento de otros incidentes que se } \\
\text { produjeran y en los que pudiera aportar conocimiento de } \\
\text { causa y efecto }\end{array}$ \\
\hline Monitorización de Incidencias & $\begin{array}{l}\text { como responsable de una salvaguarda se exige vigilancia } \\
\text { constante en su cumplimiento, debiendo reportar oportuna- } \\
\text { mente su fallo }\end{array}$ \\
\hline $\begin{array}{l}\text { Análisis y Supervisión de la } \\
\text { Medida de Control de la que } \\
\text { es responsable }\end{array}$ & $\begin{array}{l}\text { debe corroborar que la salvaguarda cumple escrupulosamen- } \\
\text { te la normativa legal y el marco de interoperabilidad en el que } \\
\text { desempeñe su trabajo }\end{array}$ \\
\hline $\begin{array}{l}\text { Manejo de Alertas } \\
\text { de Incidentes }\end{array}$ & Capacidad de Respuesta Informada de su actuación conjunta \\
\hline
\end{tabular}

\section{OBSERVACIÓN DEL CONSENTIMIENTO INFORMADO}

Conforme al Convenio de Oviedo de $1997^{10}$ propuesto por el Consejo de Europa se establece en su art. 5 que una intervención en el ámbito de la sanidad tan solo podrá efectuarse después de que la persona afectada haya emitido su expreso consentimiento, consentimiento que por otra parte podrá ser retirado en cualquier momento.

A continuación y sin la consideración esta del paciente, por ejemplo, en situaciones de "urgencia» y desde el punto de vista médico podrá ser llevada a cabo cualquier intervención en favor de la salud de la persona afectada. Debiendo, no obstante, observarse los deseos anteriores del paciente si así constara, art. 9.

9 RD 994/1999 (art. 2), RD 03/2010 (Anexo 2.4).

10 Propuesto por el Consejo de Europa establece en su art. 5 que una intervención en el ámbito de la sanidad tan sólo podrá efectuarse después de que que la persona afectada haya emitido su expreso consentimiento, consentimiento que por otra parte podrá ser retirado en cualquier momento. Es el primer instrumento internacional con carácter público vinculante para los países que lo subscriben. De manera que, el derecho a la protección a la salud queda reforzado en el terreno de la documentación clínica. 
Toda persona tiene derecho a que se respete su vida privada respecto a la información en materias de salud, respetándose en cualquier caso, la voluntad del paciente a no ser informado.

El art. 23 reconoce la contravención precisamente de estos derechos o principios garantizando una protección jurisdiccional ${ }^{11}$ de los preceptos que propone. $\mathrm{Y}$ es su art. 26 el que no admite restricción a tales derechos establecidos.

El paciente podrá manifestar en el «documento de instrucciones previas» su voluntad manifiesta con objeto de que esta se cumpla en el momento en que llegue a situaciones en cuyas circunstancias no sea capaz de hacerlo personalmente o bien una vez llegado el fallecimiento sobre el destino de su cuerpo o de los órganos del mismo. Este derecho reconocido en el art. 11 que se otorga al paciente puede designar, además, un interlocutor que mediara entre él y el médico pertinente o equipo sanitario. Creará, por consiguiente, el Ministerio de Sanidad y Consumo el Registro Nacional de Instrucciones Previas, previo acuerdo del Consejo Interterritorial del Sistema Nacional de Salud.

Por su parte la Ley L 11/2007 reconoce al ciudadano el derecho a manifestar consentimiento, pudiendo este emitirse y recabarse también por medios electrónicos.

Casos más complejos que incluso deberían poder justificarse judicialmente nos lo presenta la Agencia de Protección de Datos en su «Informe 488/2004» planteando, por ejemplo, la tesitura de la comunicación de los datos a otro facultativo de la misma especialidad que una consultante, motivada por el cese de su actividad.

Debe recordarse, nos recuerda su argumento, que de la interpretación del ya mencionado art. 17.1 y del art. 18.1 de la Ley, que dispone que «El paciente tiene el derecho de acceso, con las reservas señaladas en el apartado 3 de este artículo, a la documentación de la historia clínica y a obtener copia de los datos que figuran en ella», se desprende que los datos sólo podrían ser comunicados a otros facultativos en caso de que los mismos fueran a realizar una actividad de diagnóstico o tratamiento del paciente o el propio paciente solicitara la transmisión de su historia a su nuevo médico, sin perjuicio del deber de conservación del anterior.

La Ley, por otra parte, no impide otros usos posteriores de los datos médicos, sin perjuicio de que se introduzcan salvaguardias

${ }^{11}$ D 95/46/CE (ar. 10), LOPDAT (art. 6),L 41/2002 (art. 3 y rt. 8), RD 223/2002 (art. 6.2), L 11/2007 (a.22). 
para garantizar la confidencialidad del paciente. Así, en su art. 16.3 se contempla el acceso a la historia clínica con fines judiciales, epidemiológicos, de salud pública, de investigación o de docencia, siendo obligado preservar los datos de identificación personal del paciente, separados de los de carácter clínico-asistencial, de manera que como regla general quede asegurado el anonimato, salvo que el propio paciente haya dado su consentimiento para no separarlos. Se exceptúan los supuestos de investigación de la autoridad judicial en los que se considere imprescindible la unificación de los datos identificativos con los clínico-asistenciales, en los cuales se estará a lo que dispongan los jueces y tribunales en el proceso correspondiente.

Existe una necesidad de consentimiento expreso, aunque no necesariamente escrito para los datos relacionados con la salud, el origen racial y la vida sexual.

En caso de datos no especialmente protegidos se le otorga al afectado un plazo de treinta días para manifestar su tratamiento.

La solicitud de consentimiento respecto de un tipo de datos no podrá ser nuevamente ejecutada en el plazo de un año a contar desde la fecha de su solicitud. Tanto el derecho de acceso como el otorgamiento del consentimiento informado prescriben al de un año.

No será necesario el consentimiento del interesado en caso de urgencia relativa a la salud o para la realización de estudios epidemiológicos en los casos legalmente previstos.

En casos de trasferencias internacionales, el consentimiento informado se hace necesario para establecer la relación contractual entre el afectado y el responsable del fichero, debiéndose, no obstante:

- notificar a la Agencia Española de Protección de Datos

- e informar al interesado

Históricamente el consentimiento informado tiene su origen en el denominado Código de Núremberg ${ }^{12}$.

De acuerdo con el Código de Buenas Prácticas Clínicas ${ }^{13}$ tanto las entrevistas del consentimiento informado como su expresión escrita debe contener las siguientes indicaciones de alguna forma:

12 Código de Nuremberg.

http://www.pcb.ub.edu/bioeticaidret/archivos/norm/CodigoNuremberg.pdf (Acceso URL: julio 2012).

${ }^{13}$ Código de Buenas Prácticas Clínicas. CPMP/ICH/135/95, (pág. 18). 
- que el estudio implica investigación

- el propósito del estudio

- el acuerdo del estudio

- procedimientos a seguir

- responsabilidades del sujeto

— indicaciones de aquellos aspectos del estudio que resultan experimentales

- razonables riesgos que se prevén para el sujeto

- los beneficios esperados

- procedimientos alternativos del tratamiento

- compensaciones por perjuicios

— indicación del pago prorrateado, en su caso, por la participación

- la posibilidad de abandonar en cualquier momento el ensayo

- la identificación de la responsabilidad de la confidencialidad del sujeto en el acceso a sus datos

- duración

- número de sujetos

Un paciente puede precisar requerir el conocimiento de determinada información acerca de su historia clínica para la aplicación del tratamiento médico correspondiente que se le decida aplicar en una determinada zona geográfica de nuestro planeta. La contextura del Centro donde se pueda producir este hecho puede ser de carácter público o privado.

Cualesquiera fuera la naturaleza jurídica de los casos, se precisará la observación de la colaboración de la aplicación de la legislación de los países origen y demandante del servicio informático. Entre ambos cuerpos y apoyándose en la dinamización internacional entre ambos que se hubiera decidido existirán, además, normalizaciones y regulaciones de Organismos reconocidos que en el trascurso de la dialéctica se hubiera decidido aplicar como garantes y acompañantes en su desempeño.

El nivel de acuerdo decidido desde el Gobierno de ambos países decidirá el grado de traba a la hora de proporcionar dicha información y el formato en que será recibido por parte del Centro de Atención Médica. 
Habida cuenta de la posibilidad de Acceso por parte del ciudadano de la información necesaria acerca de su Historia Clínica, se le puede plantear al mismo el ejercicio o la determinación de sus propios criterios de filtrado de su información en la medida contemplada por la Ley General de Sanidad que se le aplicara. Tal derecho es reconocido como ejercicio de los Derechos $A R C O^{14}$, esto es, de Acceso, Rectificación, Cancelación y Oposición, resumiendo en cierto modo la defensa que le está permitida en materia de protección de datos.

Tanto es más cuando se puede ver ampliada esta perspectiva de aplicabilidad con fines de estudio de carácter estadístico. Y, de igual modo, qué ocurre en el desarrollo de la intervención médica con el manejo de los Consentimientos Informados, idénticamente se puede aplicar el concepto del tratamiento de su Historia Clínica, $H C$, en el contexto de la e-Administración.

Ya en último término se podrá provocar el bloqueo de datos o cancelación, quedando esta información tan sólo a disposición de la Administración Pública, Jueces y Tribunales. Quedando siempre amparado el paciente ante la Agencia de Protección de Datos.

El derecho de acceso contempla el conocimiento del fin último para el que se estuviera desestimado un tratamiento de datos, pudiendo ejercerse en su caso un derecho de oposición, y conocimiento exacto de las características del fichero en el que se encuentra invocado.

Los accesos restringidos podrán obtenerse por escrito, y por visualización de pantalla de ordenador .

Existen otros derechos considerados en relación a la seguridad del paciente dentro en el contexto de la e-Sanidad si tenemos en cuenta el modo en que todo ciudadano es destinatario de una Tarjeta Sanitaria Electrónica ${ }^{15}$ en la que reside una identificación digital y por medio de la cual puede identificarse en el marco de su sistema sanitario con revocación de firma electrónica.

En este punto son las Comunidades Autónomas las que se hacen responsables tanto de la tramitación de la tarjeta como de las autentificaciones realizadas en el contexto de la operativa, debiendo proporcionar idénticamente un Servicio de Atención al Cliente.

Por otra parte y dependiendo del desarrollo que haya alcanzado la aplicación de la Telemedicina en su Comunidad Autónoma, podrá

${ }^{14}$ LOPDAT (art. 5.1.d), L 41/2002 (art. 18), L 56/2007 (art. 1.d).

${ }^{15}$ L 16/ 2003, cap. V ( a.57). 
disponer de otra serie de dispositivos médicos como localizadores, servicios de domótica, pdas, etc.

La interoperabilidad entre Comunidades Autónomas permite al usuario de la e-Sanidad el acceso y obtención de informes clínicos determinados a fin de ser almacenados en dispositivos electrónicos, no considerando necesaria la citación de las copias impresas por considerarlas siempre hábiles y obvias.

Se encuentra el ciudadano con la posibilidad de vetar el acceso de determinados informes entre Comunidades y de la utilización de un sistema de alertas que le indique siempre cuál es el curso de sus diligencias incluidos las denegaciones del derecho que hubiera podido recibir.

El modelo de acceso a esta tipo de información deberá encontrarlo habilitado las veinticuatro horas del día y a lo largo de toda la semana.

Una tercera clasificación de los derechos del ciudadano se puede presentar desde el punto de vista de los Sistemas de Prevención Laboral como puede ser el caso de las Mutuas y Aseguradoras, del que siempre se deberá poder obtener el «prorrateo» del seguro que le ampara y aquel "Código Tipo» al que se encuentra adscrito un Promotor en un Sistema de Vigilancia Médica o Farmacovigilancia independientemente de que se produzca el tratamiento de datos de su HC fuera o dentro del país donde reside.

El sujeto participante en el marco sanitario también conocido como Trial Subject, puede por su parte, adoptar otra posición respecto de la defensa de sus derechos, haciéndose consciente que ni el investigador ni ningún otro miembro de la plantilla del centro sanitario donde se le deben respetar los derechos como ciudadano de la Unión Europea debieran influir en la decisión de su continuidad en el estudio o tratamiento.

No parece justo realizar una desligazón entre la figura del consumidor y el proceso concreto de generación y tratamiento de los consentimientos informados, puesto que entraña una responsabilidad manifiesta por parte del paciente no sólo el ejercicio de los Derechos ARCO sino la participación completa en el proceso de asistencia sanitario con las correspondientes afirmaciones o denegaciones de las fases de toda asistencia.

Por otra parte, podremos analizar la oportunidad de las coberturas que el Estado ha querido otorgar al papel del consumidor como 
usuario del servicio sanitario informático que le ofrece la Sociedad en la que vive.

Manifestamos las siguientes caracterizaciones que pueden llegar a condicionar la figura del consumidor en el ámbito de la e-Sanidad:

- el ejercicio de los Derechos Arco.

- por el ejercicio de los derechos a Sanidad y derechos de las Telecomunicaciones. 